\title{
Clinical implications of prenatal diagnosis of aorto-left ventricular tunnel on postnatal treatment and final outcome
}

\author{
Jovan Kosutic ${ }^{1,2}$, Sergej Prijic ${ }^{1,2}$, Mila Stajevic ${ }^{1,2}$, Marija Kalaba ${ }^{1}$, Sanja Ninic ${ }^{1}$, Zeljko \\ Mikovic $^{2,3}$, Ana Vujic ${ }^{4,5}$, Sasa Popović ${ }^{1}$ \\ ${ }^{1}$ Cardiology and Cardiac Surgery Department, Mother and Child Health Institute of Serbia, ${ }^{2}$ School of Medicine, University \\ of Belgrade, ${ }^{3}$ Obstetrics and Gynaecology Clinic Narodni Front, ${ }^{4}$ Pediatric Clinic, Clinical Centre Kragujevac, ${ }^{5}$ School of \\ Medicine, University of Kragujevac Serbia, Belgrade. \\ E-mail:sergej2804@gmail.com,kosutic@eunet.rs
}

Received: 25th November 2016, Accepted: 31st January 2017

SUMMARY: Kosutic J, Prijic S, Stajevic M, Kalaba M, Ninic S, Mikovic Z, Vujic A, Popović S. Clinical implications of prenatal diagnosis of aorto-left ventricular tunnel on postnatal treatment and final outcome. Turk J Pediatr 2017; 59: 342-344.

There are no more than 20 antenatally diagnosed aorto-left ventricular tunnel cases reported in the literature. In most of them the diagnosis was made indirectly and only after multiple fetal scans based on findings such as thick and dilated left ventricle and grossly dilated ascending aorta. We present a patient in whom a direct tunnel visualization and aorto-left ventricular tunnel diagnosis was made at the $30^{\text {th }}$ gestation week after a single fetal scan using the recently introduced "cockade sign". Clinical implications of antenatal diagnosis on postnatal treatment and outcome are also discussed.

Key words: tunnel, fetus, newborn, heart failure.

Aorto-left ventricular tunnel (AVLT) is a very rare abnormal congenital extracardiac communication between ascending aorta and the left ventricle. Its incidence ranges between 0.001 and $0.1 \%$ of all congenital heart defects seen postnatally but is much higher in prenatal heart specimens reaching $0.5 \%^{1-3}$. Nevertheless, there are no more than 20 antenatally diagnosed AVLT cases reported in the literature. In most of them the diagnosis was made indirectly and only after multiple fetal scans ${ }^{2,4}$. In only few of the reported cases diagnosis was made by direct tunnel visualization.

We present a AVLT patient in whom a correct diagnosis was made after a single scan using the recently introduced "cockade sign" 5 . Clinical implications of antenatal diagnosis on postnatal treatment and outcome are discussed. Publication is approved by Mother and Child Health Institute Ethics Committee (informed consent was received from the family).

\section{Case Report}

Mother's previous obstetric history was uneventful. This was the first, controlled pregnancy in a 26 year old woman and the first two trimester obstetric fetal scans were deemed normal. A ventricular septal defect was suspected on a regular obstetric fetal scan done in the $30^{\text {th }}$ gestation week. Expert fetal echocardiography was done 2 days later and a dilated left ventricle with dilated ascending aorta was found. The outflow tract showed two outlets: a competent but bicuspid aortic valve and, anteriorly located, a separate abnormal tunnel connecting the left ventricle with ascending aorta. The presence of the tunnel was confirmed in both the short axis and long axis view as a "double ring around the aortic annulus ("cockade sign") and severe AR through the tunnel was confirmed on color Doppler (Fig. 1. a,b,c,d). There was no VSD, pericardial effusion and or signs of fetal hydrops. The pregnancy was followed-up closely and the female newborn was spontaneously delivered at 38.5 weeks of gestation. Birth weight was $3570 \mathrm{~g}$. The baby was transferred to a pediatric hospital with cardiology/cardiac surgery facilities when she was 3 hours old. On admission, echocardiographic examination confirmed the antenatal diagnosis. There was enlarged and thick left ventricle with 
mildly reduced systolic function (LVEDD 26 mm (z-score 2.91), FS 27\%). Aortic valve was bicuspid, dysplastic without stenosis or regurgitation. Ascending aorta was significantly dilated [14.5 mm (z-score 4.51)]. There was a tunnel connecting THE ascending aorta (above the right aortic sinus of Valsalva) and the left ventricle with significant paravalvular regurgitation (Fig. 1. e,f). A large patent arterial duct was also found. After an unremarkable postnatal adaptation, the newborn, despite aggressive anti-congestive therapy, quickly developed signs and symptoms of congestive heart failure (CHF) and progressive LV dilatation with reduced contractility. Surgical procedure was performed at the age of 9 days. The procedure was done by a surgeon experienced in dealing with complex neonatal congenital heart defects.

On surgery, a diagnosis of a tunnel connecting the right aortic sinus above the sino-tubular junction with the LV was confirmed. A competent bicuspid aortic valve with severe degeneration and nodular changes of the right coronary (tunnel's) cusp was also found. Despite the gentle handing, an attempt to enter the aortic tunnel orifice with a probe resulted in the right aortic cusp disruption. It was then noted that the parietal part of this cusp was actually a part of the tunnel wall and, thus, this maneuver lead to "unroofing" of the tunnel. The augmented cusp was reimplanted to aortic annulus but with a severe post-surgery valvular aortic regurgitation (AR) (Fig. 1. g, h). Condition of the baby progressively deteriorated with severe congestive heart failure and signs of early systemic infection. A Ross procedure was discussed but not performed due to ongoing complications and eventually, eight weeks after the surgical procedure, the baby died.

\section{Discussion}

Because it is so rare, a correct AVLT diagnosis can be elusive not only in fetuses but also postnatally ${ }^{6}$. Until recently, prenatal AVLT diagnosis was mostly based on indirect signs such as LV enlargement and hypertrophy and ascending aorta dilatation and regurgitation and was made only after multiple fetal scans ${ }^{2,4}$. Direct tunnel visualization was presented only few of the reported cases ${ }^{8,9}$. Recently, a "cockade sign" - a double ring around the aortic annulus was introduced as a diagnostic clue for a correct fetal AVLT diagnosis ${ }^{5}$. We found that sign very useful and were able to make a correct diagnosis after a single fetal scan. Being so rare, we feel that whenever a dilated ascending aorta and AR are found one should think of a tunnel and search for a "cockade sign". One should bear in mind that a fetal valvular AR is extremely rare. Once a cockade sign is found, direct tunnel visualization in the long axis view with both the aortic and ventricular orifices should not be so difficult ${ }^{5}$.

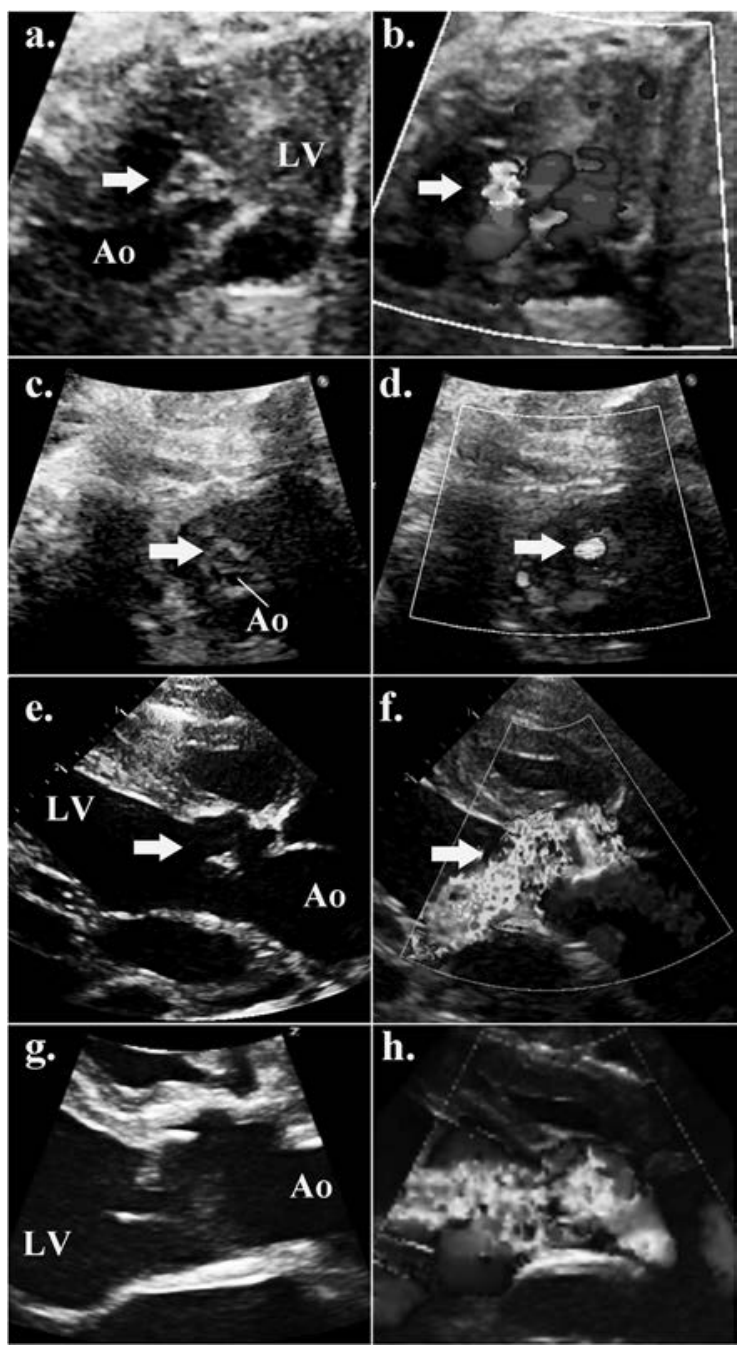

Fig. 1. Cockade sign in the long axis and short axis fetal scans - tunnel (arrows) is clearly seen with dilated ascending aorta and paravalvular regurgitation ( $\mathrm{a}, \mathrm{b}, \mathrm{c}$, d); First post birth day tunnel (arrows) parasternal long axis scan with severe tunnel regurgitation and competent aortic valve (e, f); Post-surgical parasternal long axis images - the tunnel is lost and there is severe valvular aortic regurgitation $(\mathrm{g}, \mathrm{h})$. Ao: aorta; LV: left ventricle. 
Advantages of a correct antenatal diagnosis are well known $n^{4,7}$. In our case a correct prenatal diagnosis enabled us immediate post birth patient transfer at the age of only 3 hours from a maternal Hospital to a pediatric Institution with cardiology/ cardiac surgery facilities and immediate institution of anticongestive therapy. In spite of that intractable CHF rapidly progressed. It is well known that that, in cases with significant CHF, surgical closure should be done immediately in order to prevent or minimize the development of $\mathrm{AR}^{4}$. Without surgical intervention, most children die early in infancy due to $\mathrm{CHF}^{1}$. This was exactly the situation in our patient and we opted for early surgical correction which was performed on the $9^{\text {th }}$ day of life unfortunately with unfavorable final outcome. Similar experience was described by Pascoli et $\mathrm{al}^{10}$. It seems that our case, in spite of the fact that in utero there were no signs of hydrops, was at the worse end of anatomic spectrum of AVLT patients with poor outcome ${ }^{4}$. Contrary to our two other AVLT patients, CHF in this baby could not be controlled medically. Another hypothetical explanation for the bad outcome might be a possible pre-existing aortic wall delamination which, when the probe was placed into the aortic tunnel end, resulted in ascending aorta fusion with the tunnel. Aortic wall delamination as a possible etiology of ALVT was first speculated by Cooley ${ }^{9}$ who found mucopolysaccharide deposits in aortic tunnel wall and adjacent ascending aorta ${ }^{9}$. Such tunnel histology was not confirmed by others. Aortic wall histology in our case was not done but, similar to others, we noticed disproportionate ascending aorta dilatation on our fetal scan favoring the position that the genetic origin of this condition should be searched for in the future ${ }^{1,2}$.

\section{REFERENCES}

1. McKay R. Aorto-ventricular tunnel. Orphanet J Rare Dis 2007; 2: 41.

2. Cook AC, Fagg NL, Ho SY, et al. Echocardiographicanatomical correlations in aorto-left ventricular tunnel. Br Heart J 1995; 74: 443-448.

3. Zyla-Fryczs M, Lapa P, Baranowska A, et al. An aorticleft ventricular tunnel with aortic stenosis: Diagnosis based on echocardiography in neonate. Cardiol J 2007; 14: 193-197.

4. Sousa-Uva M, Touchot A, Fermont L, et al. Aortico-left ventricular tunnel in fetuses and infants. Ann Thorac Surg 1996; 61: 1805-1810.

5. Christmann M, Dave H, Buechel EV. Prenatal diagnosis of aorto-left ventricular tunnel: introducing the 'cockade signe'. Eur Heart J 2015; 36: 1136.

6. Pockett CR, Chan S, Smallhorn J. Aortico-left ventricular tunnel: The elusive diagnosis. Pediatric Cardiol 2013; 34: 1743-1745.

7. Kolcz J, Januszewska K, Malec E. Successful repair of aorto-left ventricular tunnel diagnosed prenatally. Cardiol Young 2005; 15: 219-222.

8. Singh A, Reinhardt Z, Desai T, Rasiah SV. Case series of antenatally diagnosed aortico-left ventricular tunnel. Pediatr Cardiol 2011; 32: 822-825.

9. Cooley RN, Harris LC, Rodin AE. Abnormal communication between the aorta and left ventricle: aortic-left ventricular tunnel. Circulation 1965; 31: 564-571.

10. Pascoli I, Cester M, Nanhorngue K, et al. Aortico-left ventricular tunnel diagnosed prenatally: case report. Prenat Diagn 2007; 27:1263-1265. 